4 AI, aged between 5-17 years old, across four national specialistled units: Eastman Dental Hospital, Guy's and St Thomas' Hospitals, Birmingham Dental Hospital and the Leeds Dental Institute. The PROM questionnaire was tailored to the patients' symptoms, condition and quality of life. Interestingly, this method allowed the patients to express their own views on how they felt about having dental treatment; whether they were happy with the appearance of their teeth, the physical symptoms they were experiencing and the impact AI had on their day-to-day lives.

Overall, $72 \%$ of the patients participating expressed that they often or occasionally experienced pain or sensitivity. Additionally, $76 \%$ of patients that participated reported that they often or occasionally felt unhappy with the way their teeth looked, with a minority of patients experiencing teasing or bullying by their peers and having a lack of confidence to smile. Reassuringly, as treatment progressed, $81 \%$ of patients indicated that they were happy with the appearance of their teeth - a notable contrast from the $33 \%$ of patients that were happy pre-treatment.

Using a PROM questionnaire in this study highlighted not only the aesthetic concerns that CYP face with AI, but also the impact this has on their overall wellbeing. Promisingly, this study emphasised how the current AI care in specialist-led centres across the UK has a positive effect on the patients' views on their own dentition. This study has made it evident that using a simple PROM questionnaire throughout treatment planning helps to address patients' concerns, which in turn can provide a more effective, holistic approach to AI care in CYP.

By Rushali Lakhani

Third Year Dental Student, University of Leeds, UK

\title{
What needs to change? The perspective of our Urgent Dental Care centre heroes
}

\section{The future of dentistry post-COVID-19: perspectives from Urgent Dental Care centre staff in England.}

BrDent J 2021; https://doi.org/10.1038/s41415-021-3405-1

The COVID-19 pandemic sent shockwaves through the world of dentistry and created the biggest disruption to dental services within our lifetimes. Furthermore, the effect of this virus has exacerbated several existing issues and created several new conundrums facing NHS dentistry.

This research compiled the views of 29 dentists and nine dental nurses who worked in Urgent Dental Care centres between June and August 2020. Through the interviews, five themes were identified as needing to be addressed to ensure the feasible future of NHS dental services.

The first theme looked into the backlog of patient care and access issues. Interviewees were alarmed about an already difficult-to-access system becoming even harder to access. Concern was also raised about waiting lists for secondary care services. Consequences of access issues included delayed diagnoses and increased recall intervals.

Theme two explored the sustainability of dental practices, highlighting concerns about restrictions limiting patient contact and the impact COVID-19 has had on patients' finances, leading to more patients expecting NHS treatments as opposed to private alternatives. The cost of PPE and the uncertainty around Brexit added to worries about practice finances.

The 'workforce sustainability' theme identified the theory that the pressure of COVID-19 may increase the already high risk of burnout and lead to an increase in dental professionals leaving the profession. Students and foundation dentists have also had much less exposure and experience than previous cohorts, which may be 'detrimental' to the future workforce and impact patient safety.

Clinicians highlighted the 'identity of dentistry' has evolved towards relieving pain and infection, and an increased need to focus on preventative measures. A suggestion was also made to align dentistry to a greater extent with general healthcare, due to the 'unique' role dentists have in addressing public health issues such as obesity.

The most frequently raised theme identified was 'contract reform'. Dismay was evident at the current UDA-based system in the long term and during the current pandemic. A move to a 'core' service was suggested, while identifying the need to avoid disadvantaging certain groups. Particularly associates seemed to prefer a move towards a salaried contract, while a capitation-based system was deemed unlikely.

These themes highlight the current climate of NHS dentistry, which is only going to worsen unless urgent action is taken to address these concerns. It also emphasises the need for preparation to respond to the public health emergencies of tomorrow.

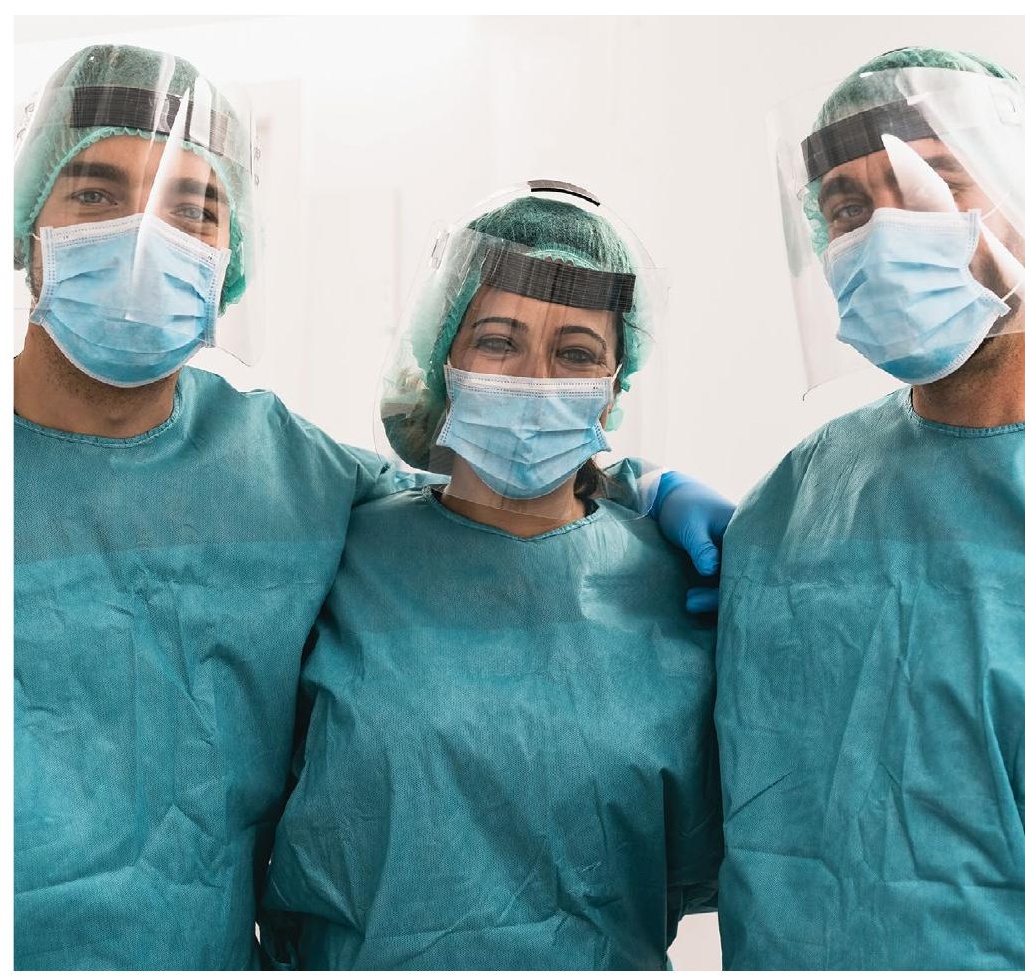

